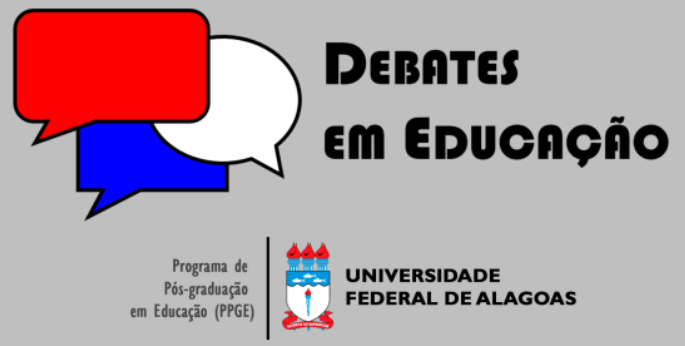

ISSN Eletrônico 2175-6600

Vol. 12 | Nº 27 | Maio/Ago. | 2020

\section{Edinéia Woiciekovski}

9

Pontifícia Universidade Católica do Paraná (PUCPR) edineia.w@hotmail.com

\section{Alboni Marisa Dudeque Pianovski Vieira}

9 iD

Pontifícia Universidade Católica do Paraná (PUCPR) alboni@alboni.com

\section{A MANIFESTAÇÃO DO "PRÊMIO DE BELEZA" NA ÁREA DE ENSINO}

\section{RESUMO}

Neste artigo, busca-se investigar se existe relação entre a beleza dos professores e a avaliação docente realizada pelos alunos, atribuindo vantagens aos professores percebidos como mais atrativos. Por meio de uma revisão de literatura, foram analisadas publicações que tratam do tema, buscando identificar se existe manifestação do "prêmio de beleza" em sala de aula e se ocorre maior incidência para um determinado gênero. Os resultados demonstraram que o nível de atratividade percebido nos professores impacta significativamente nas avaliações de ensino. Além disso, os artigos estudados revelam que, de modo geral, há maior incidência do "prêmio de beleza" para os homens, tendo as professoras mulheres recebido avaliações significativamente menores.

Palavras-chave: Prêmio de Beleza. Avaliação de professores. Gênero.

\section{THE MANIFESTATION OF A "BEAUTY AWARD" IN THE TEACHING FIELD}

\section{ABSTRACT}

In this article, we seek to investigate if there is a relationship between the beauty of the teachers and the teacher evaluation carried out by the students, attributing advantages to teachers perceived as being more attractive. Through a literature review, publications that deal with the subject were analyzed, seeking to identify if there is a "beauty award" in the classroom and if there is a greater incidence for a given gender. The results showed that the level of attractiveness perceived by teachers has a significant impact on the teacher evaluations. Furthermore, the articles studied reveal that, in general, there is a higher incidence of the "beauty award" for men, with female teachers receiving significantly lower ratings.

Keywords: Beauty award. Teacher evaluation. Gender.

Submetido em: $14 / 10 / 2019$

Aceito em: 13/04/2020

Publicado em: 22/06/2020

do: http://dx.doi.org/10.28998/2175-6600.2020v12n27p200-213 


\section{INTRODUÇÃO}

No relacionamento entre as pessoas, a aparência física possui relevante papel influenciador, uma vez que afeta o tratamento que as pessoas recebem, bem como a forma como tratam os outros (ANDREONI, PETRIE, 2008). Mesmo em um curto espaço de tempo, as pessoas fazem julgamentos automáticos sobre as características de uma pessoa com base em sua aparência facial, sendo que são rapidamente extraídas inferências acerca de sua personalidade (TODOROV, PAKRASHI, OOSTERHOF, 2009). O relacionamento existente entre alunos e professores não é diferente, pois muitos alunos, em avaliações realizadas pelas instituições de educação superior, classificam melhor os professores considerados mais atrativos, além de se verificar que há maior frequência nas aulas ministradas por esses docentes (WOLBRING, RIORDAN, 2016).

Tal fenômeno recebe o nome de "prêmio de beleza", termo cunhado por Hamermesh e Biddle (1993), e que existiria para aqueles que possuem boa aparência, admitindo-se que também existiria uma penalidade significativa para aqueles que possuem pouca atratividade, com reflexos no salário dos indivíduos. Uma das razões encontradas para esse efeito ocorre em função de que a beleza é produtiva, pois resulta na preferência dos clientes ou na contratação, em virtude da discriminação do empregador. Ainda segundo esses autores, o salário das pessoas com aparência abaixo da média é inferior ao dos trabalhadores com boa aparência.

O "prêmio de beleza" está presente em diversos contextos, tais como no rendimento dos trabalhadores (BIDDLE, HAMERMESH, 1998; SALA, et al., 20।3); no pagamento de gorjetas aos garçons em restaurantes (PARRETT, 20I5); em campanhas eleitorais BERGGREN, JORDAHL, POUTVAARA, 20 I0); e no contexto universitário (DERYUGINA, SHURCHKOV, 20I5a), além de ser associado com a saúde ( KALICK et al., 1998), com a felicidade (HAMERMESH, ABREVAYA, 20I3) e a inteligência (KANAZAWA, KOVAR, 2004).

Contudo, a atratividade nem sempre é associada com características positivas, sendo manifestada, em algumas circunstâncias, como uma "penalidade de beleza", pois, conforme Deryugina e Shurchkov (20 I5b) e Andreoni e Petrie (2008), indivíduos com bela aparência podem receber maiores punições, o que ocorre quando seu desempenho fica aquém das expectativas geradas pela aparência física (WOLBRING, RIORDAN, 2016).

No mesmo sentido, Feingold (1992) afirma que ser atrativo não está relacionado com a saúde, a inteligência e o desempenho na educação superior, pois, no experimento por ele realizado, foi encontrado efeito de relação com a atratividade apenas para a habilidade social dos indivíduos. Dessa forma, o estudo evidenciou que as pessoas atrativas não são aquilo que pensamos. 
Numa perspectiva racional, a beleza não deveria influenciar as percepções dos alunos quanto à qualidade do ensino dos professores nas avaliações realizadas pelas instituições de educação superior, nem ser um indicador da frequência dos estudantes na sala de aula. Não deveria, tampouco, ser um pressuposto de saúde, felicidade e inteligência, como muitos estudos evidenciam.

Com base no contexto exposto, o objetivo desta pesquisa é responder à seguinte pergunta: qual a relação entre o efeito da beleza dos professores e a avaliação de ensino realizada pelos alunos? Para tanto, o estudo tem como objetivo geral analisar publicações que tratam do tema "prêmio de beleza" manifestado na área de ensino, desdobrando-se nos seguintes objetivos específicos: (i) identificar se existe manifestação do "prêmio de beleza" na sala de aula expresso por meio das avaliações de ensino; (ii) verificar se o gênero dos professores foi analisado nos estudos pesquisados e se houve maior manifestação do "prêmio de beleza" para um determinado gênero.

Após esta breve introdução, o estudo está estruturado nos seguintes tópicos: seção teórica sobre o "prêmio de beleza" e sua manifestação em diversos contextos; o gênero como fator indutor de sua atribuição; resultados obtidos a partir da análise dos artigos que abordaram a questão da manifestação do "prêmio de beleza" na área de ensino, no que tange à avaliação dos professores realizada por alunos, seguindo-se as considerações finais.

\section{O "PRÊMIO DE BELEZA"}

Um tratamento diferenciado tem sido observado para as pessoas consideradas fisicamente mais belas ou atrativas, independentemente de suas habilidades ou de sua produtividade no trabalho. Em linhas gerais, são atribuídas características mais positivas às pessoas consideradas mais atrativas (EAGLY et al., 1991; LANGLOIS et al., 2000), sendo elas consideradas mais felizes e confiantes ( HAMERMESH, ABREVAYA, 2013; Eagly et al., 1991; WILSON, ECKEL, 2006).

○ "prêmio de beleza" foi primeiramente registrado e cunhado em um contexto empresarial, campo em que a atratividade se revela essencial para compreender diversos fenômenos, tais como a contratação de novos funcionários, o aumento salarial de determinados indivíduos que exercem a mesma função em detrimento de outros, bem como o relacionamento com os diversos stakeholders. Dessa forma, é imprescindível que os gestores, aqui incluídos os gestores educacionais, possuam conhecimento acerca da influência que a beleza exerce na respectiva área.

Muitos estudiosos argumentam que a preferência pela atratividade é aprendida após a exposição aos padrões existentes na sociedade. No entanto, um estudo realizado por Langlois et al. (|99|) sugere que a preferência por faces atrativas, independentemente de gênero, idade ou raça, está presente até 
mesmo em bebês, pois crianças de seis meses conseguem discriminar faces atraentes dentre outras menos atraentes, preferindo visualmente rostos atraentes.

No mesmo sentido Eagly et al. (|99|) citam, como exemplo, que a cultura americana associa a beleza a coisas boas e a ausência de beleza a coisas ruins, pois em programas televisivos e livros infantis a bruxa malvada é feia, ao passo que príncipes e princesas são bonitos. Da mesma forma, na publicidade, modelos atraentes ocupam espaços positivos, sendo bem-sucedidos em suas carreiras e possuindo bens valiosos, induzindo as pessoas a atribuírem tais aspectos para suas vidas. Assim, a característica de ser atrativo tem sido associada à beleza e à competência, como uma forma de poder e, nesse sentido, a beleza se torna um ingrediente essencial para a felicidade.

○ "prêmio de beleza" pode estar presente em muitos âmbitos, no contexto da educação superior. Deryugina e Shurchkov (20I5a) estudaram as vantagens da beleza no ingresso e durante o curso na universidade. $\bigcirc$ estudo revelou que não existem relações significativas entre tais processos, e, no que se refere ao ingresso, o candidato é avaliado por testes, sendo que a atratividade é negativamente correlacionada com a capacidade que é medida por esses testes. Não se constata, portanto, que os indivíduos mais atraentes podem ser menos inteligentes ou dedicar um esforço menor na preparação para o teste. $\bigcirc$ estudo encontrou indícios do "prêmio de beleza" somente em áreas específicas de estudo, com mulheres mais atraentes possuindo maior probabilidade de estudar na área de economia e de se tornarem consultoras, analistas ou gerentes, enquanto possuem menor possibilidade de estudar na área de ciências e trabalhar na área científica ou técnica. O padrão observado sugere que mulheres mais atraentes possuem maior autoconfiança, sendo, assim, mais propensas a se candidatar em processos seletivos nas universidades, embora não seja encontrada nenhuma evidência de que as mulheres mais atraentes tenham recebido um "prêmio de beleza" no processo de ingresso nas universidades estudadas.

Entretanto, Kanazawa e Kovar (2004) afirmam que as pessoas mais atraentes são mais inteligentes, embora muitos pesquisadores descartem essa percepção e a tratem apenas como um viés ou estereótipo. Tal afirmação resulta das suposições de que os homens mais inteligentes ocupam cargos que possuem melhor status, e homens que possuem maior status são propensos a se casar e ter filhos com mulheres mais bonitas do que homens que possuem menor status, e por fim, a inteligência e a beleza são consideradas hereditárias.

Tratando do efeito da beleza na primeira fase do processo de contratação no mercado de trabalho, Ruffle e Shtudiner (20 I 4) enviaram currículos em seis versões diferentes em resposta a anúncios publicados em Israel, sendo que metade dos currículos possuía uma fotografia de um candidato masculino ou feminino, atraente ou sem atrativos. Cada currículo que possuía a fotografia do candidato foi emparelhado com um currículo de controle idêntico, porém sem a imagem. A inclusão da fotografia no currículo teve um efeito negativo, sendo que o retorno no que se refere ao agendamento para entrevistas para currículos 
com imagem foi I,4\% menor do que para currículos sem fotografia. Entretanto, foi observado um "prêmio de beleza" para os homens, pois os currículos com fotografia de homens atraentes obtiveram uma taxa de resposta de 19,7\% em relação à taxa de resposta para currículos de homens sem imagens, que ficou em 13,7\%. Por outro lado, a taxa de resposta para as mulheres foi surpreendente: currículo sem fotografia apresentando a maior taxa de resposta, com 16,6\%; na sequência, mulheres de atração média com 13,6\%; e mulheres atraentes com 12,8\%. A penalidade atribuída às mulheres atraentes contradiz as observações em estudos anteriores observados pelos autores, pois geralmente os estudos associam a beleza com traços positivos nas pessoas.

A beleza também está relacionada com a felicidade. Hamermesh e Abrevaya (2013) sugerem que a beleza de uma pessoa aumenta sua satisfação, ou seja, sua felicidade, de forma indireta, levando-a a alcançar resultados mais desejáveis em educação, saúde e ganhos mais altos no mercado de trabalho, além de favorecer a atração de cônjuges com rendas maiores. Dessa forma, os efeitos da beleza estariam relacionados tanto com resultados econômicos como não econômicos.

A atratividade facial de um indivíduo é também um pressuposto de sua saúde: este foi o principal achado que Kalick et al. ( 1998) obtiveram na condução de uma pesquisa que utilizou fotos de 164 homens e 169 mulheres adolescentes, sendo que os dados de atratividade foram baseados nas fotografias faciais, e as condições de saúde dos indivíduos foram verificadas a partir de registros de exames médicos. Aos participantes do estudo era solicitado avaliar a condição de saúde dos indivíduos representados em cada fotografia. Os resultados demonstram evidências de que as pessoas são "cegadas" pela beleza, pois erroneamente os participantes consideraram os indivíduos mais atrativos como mais saudáveis, sejam os participantes homens como mulheres. Desta forma, embora não exista qualquer relação entre a atratividade e a saúde dos indivíduos, na percepção dos participantes avaliadores do estudo ambos foram fortemente relacionados.

Diversos estudos que abordam a temática do "prêmio de beleza" verificam a variável gênero como fator indutor ou até mesmo de distinção na atribuição do referido efeito. Em função disso, o tópico subsequente apresenta estudos que mensuraram o gênero como fator indutor do "prêmio de beleza".

\section{O GÊNERO COMO FATOR INDUTOR DO “PRÊMIO DE BELEZA"}

Hamermesh e Biddle (1993), autores que definiram a existência de um "prêmio de beleza", argumentam que, em relação ao gênero, o "prêmio de beleza" pode ser maior para os homens, embora essa diferença não seja considerada significativa.

Johnson e Mislin (20 I I) identificaram que a atratividade é benéfica tanto para homens quanto para mulheres que se candidatam para a maioria dos empregos, sendo que ser um homem atraente é benéfico 
tanto para se candidatar a um emprego predominantemente feminino quanto masculino. No entanto, o mesmo não ocorre com as mulheres, que obtiveram maiores benefícios de serem atraentes somente quando se candidatavam a empregos voltados para mulheres, revelando ainda que a atratividade poderia ser prejudicial às mulheres que se candidatavam para trabalhos masculinos nos quais a aparência física não possuía importância.

Um estudo realizado na Austrália, por Borland e Leigh (2014), evidenciou a existência de uma relação entre beleza e horas trabalhadas e, consequentemente, o salário dos indivíduos, identificando que existem diferenças entre homens e mulheres. Para as mulheres, a beleza diminui as horas trabalhadas sem alterar o salário e, para os homens, isso está associado principalmente a uma "penalidade de beleza", que é aplicada quando a pessoa é considerada atrativa abaixo da média.

A distribuição de salários pode variar de acordo com os efeitos da beleza, segundo Doorley e Sierminska (20 I5). Para esses autores, o efeito da beleza é notável, principalmente nos salários mais baixos das mulheres e para as mulheres que possuem alto nível de instrução, ocorrendo uma "penalidade de beleza" para mulheres que possuem escolaridade mais baixa. Já para os homens, o "prêmio de beleza" está presente em todas as faixas de distribuição salarial, sendo apresentado mais fortemente para aqueles que possuem maior educação. Assim, o "prêmio de beleza" é elevado para aqueles que possuem formação universitária, independentemente de gênero.

Buscando examinar se os retornos da atratividade diferem entre homens e mulheres, ou seja, se há diferenças nos salários baseadas na atratividade, Wong e Penner (20 I 6) identificaram que os indivíduos atraentes ganham aproximadamente $20 \%$ mais em relação àqueles médio atraentes, sendo que, no estudo realizado, os autores não encontraram diferença significativa de gênero nos retornos de atratividade. Em suma, a atratividade não foi considerada mais ou menos importante para as mulheres do que para os homens, mesmo que as mulheres ganhem menos do que os homens, e os indivíduos pouco atraentes ganhem menos do que os indivíduos atraentes, sendo que foi encontrado apoio para o fato de que a beleza é um fator de status e estratificação.

Em um estudo que visou decompor o "prêmio de beleza" no momento de contratação de funcionários, bem como medir a confiança depositada pelo empregador no trabalhador, Mobius e Rosenblat (2005) encontraram que, em relação à percepção de confiança, que foi obtida pela estimativa do empregador quanto à produtividade de cada trabalhador, os homens não foram considerados mais confiantes, ainda que desempenhassem a função de resolver os labirintos melhor do que as mulheres. Nesse estudo, portanto, não existiram efeitos de gênero.

Em um contexto que investigou a beleza e o sucesso eleitoral, Berggren et al. (2010) encontraram que tanto homens como as mulheres avaliam candidatos eleitos e não eleitos de forma similar. Contudo, as candidatas mulheres eleitas são consideradas pelos eleitores melhores em relação àquelas que não 
foram eleitas, sendo que os homens atribuem melhores avaliações de competência para as candidatas eleitas.

Adiante, trata-se dessa temática relativa ao prêmio e sua aplicação quanto ao gênero dos indivíduos, de modo particular, voltada para a área de ensino.

\section{MANIFESTAÇÃO DO “PRÊMIO” E DA “PENALIDADE DE BELEZA” NA ÁREA DO ENSINO}

O estudo seminal acerca da beleza na sala de aula é de Hamermesh e Parker (2005), que buscaram mensurar a produtividade do ensino dos professores de diversos departamentos e níveis hierárquicos da Universidade do Texas, nos Estados Unidos. As avaliações utilizadas no estudo foram aquelas exigidas pela universidade, realizadas sem a presença do professor, por meio de um formulário em que era possível assinalar se o ensino ministrado pelo professor durante as aulas poderia ser considerado: (i) muito insatisfatório; (ii) insatisfatório; (iii) satisfatório; (iv) muito bom e (v) excelente. Cada fotografia dos professores foi avaliada em uma escala de 10 pontos por seis alunos de graduação, sendo três homens e três mulheres. Os resultados indicaram que a beleza resultava em produtividade e que a aprendizagem era maior pelo fato de que os alunos prestavam mais atenção nas aulas dos professores considerados mais atrativos. Os autores da pesquisa questionam se os alunos discriminam os professores considerados menos atrativos ou se, realmente, aprendem menos com esses professores.

Visando a identificar se o "prêmio de beleza" se manifestaria em um contexto educacional distinto, Süssmuth (2006) aplicou o estudo de Hamermesh e Parker (2005) na Alemanha, em que - ao contrário do sistema de ensino nos EUA, que exige avaliação de todos os professores pelos alunos -, somente algumas universidades ou departamentos realizam essa avaliação. Participaram do estudo cinquenta alunos dos cursos de economia e administração da Universidade de Bamberg, que foram convidados a avaliar o nível de atratividade de cinquenta professores que não faziam parte do quadro permanente da Universidade, mas que haviam ministrado I I I cursos no inverno de 2002 e 2003 e no verão de 2003, sendo que o desempenho no ensino foi avaliado a cada semestre pelos alunos. A atratividade dos professores, in casu, foi avaliada por estudantes de uma universidade diferente, com o intuito de superar o viés de percepção de beleza por causa da reputação do professor no ambiente universitário. Os achados indicaram que a percepção de atratividade dos alunos quanto aos professores melhorou a classificação da aula em cerca de 0, I I a 0, 15. Comparando com o estudo de Hamermesh e Parker (2005) que obtiveram resultado entre 0,17 a 0,38 , o estudo realizado com estudantes alemães sugere somente cerca de metade do efeito do "prêmio de beleza" encontrado no EUA. 
Ponzo e Scoppa (2013) utilizaram dados correspondentes a um período de sete anos, relativos à avaliação de ensino dos alunos da faculdade de economia, que compreende os cursos de administração, direito, estatística, economia e ciências sociais da Universidade de Calabria, localizada no Sul da Itália, durante o qual foram avaliados 234 professores de 2.512 cursos ministrados na instituição. $\bigcirc$ estudo controlou diversas variáveis dos professores, como gênero, idade, dedicação integral ao exercício da docência, além de outras relativas ao curso. Os resultados demonstraram que a beleza dos professores possuía forte impacto nas avaliações realizadas pelos alunos, sugerindo que existe discriminação em favor daqueles que possuem melhor aparência, mesmo tendo sido controladas todas as variáveis mencionadas. No que diz respeito à idade dos alunos, a beleza é considerada mais importante para os alunos homens do $1^{\circ}$ e $2^{\circ}$ anos do curso, sendo que aos poucos a relevância é diminuída.

Em estudo realizado por Wolbring e Riordan (20 I 6), foi possível perceber que os alunos classificam melhor os professores atrativos, em uma avaliação denominada "student's evaluations of teaching", além de frequentarem mais as aulas desses professores. Entretanto, os professores também são avaliados de acordo com seu comportamento e, quando se desviam das expectativas dos alunos, o efeito de atratividade pode se tornar negativo, transformando o prêmio em uma penalidade, sendo os professores mais belos sancionados quando realizaram um teste rígido. Esse resultado foi obtido em um estudo conduzido na Universidade de Munique, em que os alunos receberam um currículo dos professores incluindo uma fotografia e ouviram um breve áudio de uma palestra gravada pela pessoa do currículo. Ao final, foram convidados a fornecer uma classificação e realizar um teste sobre a palestra, sendo que os professores atraentes que aplicaram um teste mais difícil foram punidos severamente na classificação da palestra. Em suma, o estudo revelou que o "prêmio de beleza" pode se transformar em uma penalidade sob certas circunstâncias, e que a aparência dos professores resulta em tratamentos distintos.

Além de estudar a percepção dos estudantes quanto à atratividade física dos professores, Mendez e Mendez (2016) incluíram percepções quanto à idade dos professores que compõem o corpo docente, seus conhecimentos e acessibilidade, argumentando que a percepção de atratividade pode afetar até mesmo a preferência dos alunos por determinada universidade, no momento de escolha. $\bigcirc$ estudo conduzido envolveu duas pesquisas em uma universidade nos EUA e os estudantes participantes estavam matriculados em uma disciplina obrigatória a todos os cursos de graduação, com o objetivo de não limitar a amostra a um determinado curso. Aos estudantes foi solicitado que avaliassem uma série de fotografias de professores de universidades fora do estado em que a pesquisa ocorreu, sendo que na primeira pesquisa foi solicitado avaliar a idade e atratividade e na segunda o conhecimento e acessibilidade dos professores. Os achados desse estudo apontaram, de modo geral, que a atratividade física do corpo docente influencia a percepção dos estudantes, os quais atribuem aos professores mais jovens e atraentes maior acessibilidade; por outro lado, os professores mais velhos foram considerados mais experientes. 
Além disso, os estudantes preferem realizar disciplinas que possuem professores atraentes e acessíveis, independentemente do conhecimento percebido.

Os estudos analisados nesse artigo utilizaram uma determinada quantidade de fotos, conforme consta do Quadro I.

Quadro I - Quantidade de fotos analisadas nos estudos
\begin{tabular}{|c|c|}
\hline Hamermesh e Parker (2005) & 94 \\
\hline Sussmuth (2006) & 50 \\
\hline Ponzo e Scoppa (2013) & 234 \\
\hline Mendez e Mendez (2015) & 144 \\
\hline Wolbring e Riordan (2016) & 125 \\
\hline
\end{tabular}

Fonte: os autores (2017)

O Quadro I apresenta a quantidade de fotos de professores utilizada em cada estudo analisado nesse artigo. Cada fotografia foi submetida ao processo de avaliação do nível de atratividade a fim de ser utilizada no estudo. Dessa forma, é possível verificar disparidade entre os números, que são variáveis em função do período de análise bem como do total de aulas ministradas.

No entanto, os estudos analisados possuem algumas peculiaridades, conforme a Figura I, na qual é possível verificar semelhanças expressas na frequência com que determinadas palavras foram repetidas no título, resumo e palavras-chave de cada trabalho.

Figura I - Palavras mais frequentes nos artigos analisados

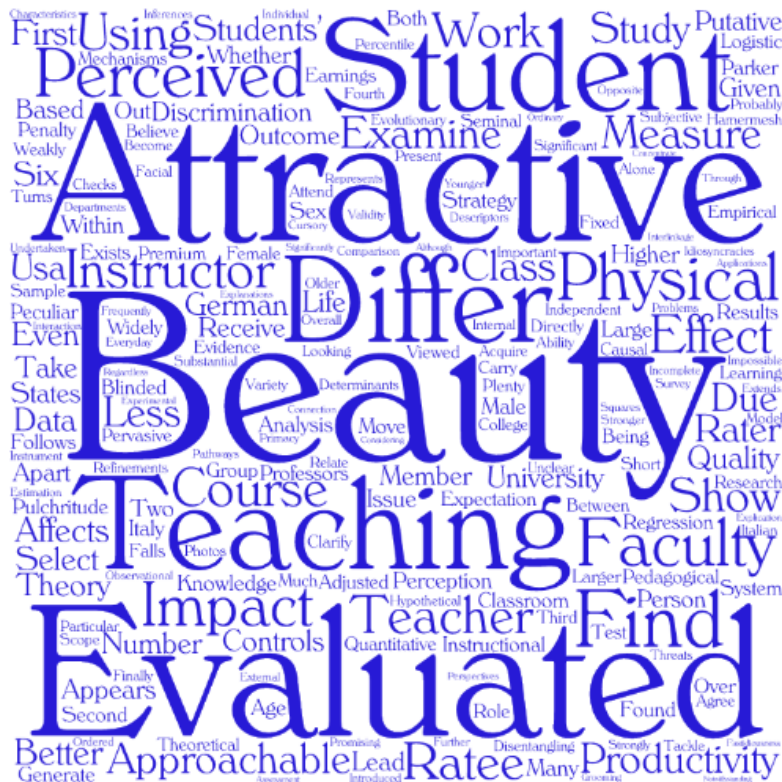

Fonte: desenvolvido por meio do site https://tagul.com/create, 2017, pelos autores. 
Conforme a Figura I, as palavras mais frequentes são Beauty e Attractive, seguido de Evaluated e Teaching, demonstrando exatamente o propósito deste artigo em analisar trabalhos acerca da beleza dos professores na área de ensino, expressa nas avaliações realizadas pelos alunos.

O próximo item discute se a variável gênero foi controlada e analisada nos artigos em questão.

\section{O GÊNERO DOS PROFESSORES COMO FATOR DE ATRIBUIÇÃO DO “PRÊMIO DE BELEZA"}

No estudo seminal realizado por Hamermesh e Parker (2005), as professoras (gênero feminino) receberam classificações significativamente menores, o que evidenciou que o efeito do "prêmio de beleza" ocorre para os professores (gênero masculino), mostrando haver relação entre o gênero do professor e sua avaliação de ensino. A fim de explorar essa diferença de gênero os autores definiram novo modelo separando por gênero, sendo que os resultados apontam que o impacto da beleza para as mulheres é de fato menor. Já para os professores homens o prêmio de beleza se manifesta de forma positiva, porém pode se revelar como uma penalidade. Hamermesh e Parker (2005) consideraram que a composição de gênero dos estudantes pode ser alterada de acordo com o curso, e que alguns cursos podem atrair estudantes com percepções distintas com relação à beleza, além disso os diferentes graus de dificuldades de cada curso podem influenciar a classificação dos professores. Essas possibilidades foram testadas pelos autores nesse estudo, sendo que o impacto da beleza foi um pouco menor do que nas outras estimativas, mas ainda assim é considerado robusto.

$\mathrm{Na}$ aplicação da pesquisa de Hamermesh e Parker (2005) realizada por Süssmuth (2006), na Alemanha - país com diferenças no sistema educacional em relação aos EUA, foram verificadas questões de gênero, tendo como resultado, na amostra total, que as professoras foram classificadas significativamente pior em comparação com os professores. No entanto, esse estudo revela que a idade dos estudantes participantes da pesquisa impactou na percepção de atratividade dos professores, sendo que entre os mais jovens existe maior discriminação com relação à atratividade. Alguns indícios, entretanto, apontam que a distinção por gênero dos professores ocorre principalmente entre os estudantes de cursos mais avançados.

No contexto universitário italiano, Ponzo e Scoppa (2013) revelaram que a beleza dos professores é extremamente significativa quanto às avaliações dos alunos, sendo que, em relação ao gênero, encontraram que a beleza é mais importante para as mulheres do que para os homens. Dessa forma, as professoras (gênero feminino) tendem atrair avaliações piores em relação aos professores. Porém, na investigação separando os grupos por gênero, os resultados demonstram que a beleza é estatisticamente significativa para os dois gêneros. 
Os professores do gênero masculino foram percebidos como mais experientes em relação às professoras do gênero feminino, fato relevado em pesquisa realizada por Mendez e Mendez (20।6), que afirmaram que professoras enfrentam obstáculos mais desafiadores na sala de aula do que professores. Além disso, o próprio gênero dos alunos mensurou os professores de forma distinta, sendo que os estudantes do gênero masculino avaliaram os professores como "menos atraente", e os estudantes do gênero feminino como "mais atraente". Em todas as categorias analisadas nesse estudo (acessibilidade, conhecimento, entre outros), as professoras foram classificadas com notas mais baixas pelos alunos, além de receber mais comentários negativos.

Wolbring e Riordan (2016) afirmam que a penalidade imposta aos indivíduos é especialmente maior para os homens professores, visto que as mulheres recebem um "prêmio de beleza" que compensa parcialmente sua penalidade. No entanto, um dos principais achados do estudo revela que, tanto homens quanto mulheres, considerados mais atrativos, são punidos severamente por suas falhas.

Como é possível observar, a variável gênero foi analisada pelos artigos estudados nessa revisão de literatura, sendo possível perceber que, de modo geral, há maior incidência do "prêmio de beleza" para os professores homens, pois as professoras mulheres receberam avaliações significativamente menores.

\section{CONSIDERAÇÕES FINAIS}

O "prêmio de beleza", termo cunhado por Hamermesh e Biddle (1993) e que teve seu efeito registrado primeiramente em um contexto empresarial, pode se manifestar em diversas áreas. Na área de ensino não é diferente: a simples visualização das fotografias dos professores no site das instituições de educação superior, no momento da escolha de um determinado curso, pode ser influenciada pelo efeito da beleza dos professores. Esse efeito pode interferir também no relacionamento entre estudantes e professores em sala de aula, impactando inclusive na frequência dos alunos às aulas.

Este estudo teve por objetivo realizar uma revisão de literatura com artigos que tratam da temática do "prêmio de beleza" registrado nas avaliações do corpo docente realizadas pelos estudantes. Os achados de tais estudos revelaram que o nível de atratividade percebido dos professores impacta significativamente nas avaliações de ensino, refletindo até mesmo na aprendizagem dos alunos, que pode ser considerada maior pelo fato de eles prestarem mais atenção nas aulas dos professores considerados mais atrativos.

O estudo da variável gênero, constante dos artigos analisados, levou à percepção de que as mulheres não recebem a mesma consideração, em relação ao "prêmio de beleza", que os homens, que nesse particular são favorecidos.

Uma das limitações desse estudo foi analisar a incidência do prêmio de beleza na avaliação de professores em outros países, sem realizar uma investigação da realidade brasileira. No entanto, nosso 
intuito de analisar publicações que tratam do tema "prêmio de beleza" manifestado na área de ensino foi cumprido, pois não encontramos publicação dentro desse tema no Brasil.

○ "prêmio de beleza" na área de ensino foi analisado em diversas universidades de diferentes países, sendo que não foi encontrada publicação dessa natureza no Brasil. Dessa forma, sugere-se a aplicação desse estudo em uma universidade brasileira, a fim de se verificar a incidência de tal efeito. Nesse sentido, é necessário construir parâmetros tendo em vista o contexto do Brasil, sendo necessário incluir fatores como a classe social, raça, e o gênero como apresentado, que podem impactar nos resultados a ser encontrados. Nossa sugestão para construção desse estudo no Brasil é utilizar como parâmetro o estudo seminal de Hamermesh e Parker (2005) devido a relevância e contribuição desse artigo dentro da temática do prêmio de beleza aplicado à área de educação e avaliação de professores.

\section{REFERÊNCIAS}

ANDREONI, J.; PETRIE, R. Beauty, gender and stereotypes: Evidence from laboratory experiments. Journal of Economic Psychology, v. 29, n. I, p. 73-93, fev. 2008.

https://doi.org/l 0.1016/j.joep.2007.07.008

BERGGREN, N.; JORDAHL, H.; POUTVAARA, P. The looks of a winner: Beauty and electoral success. Journal of Public Economics, v. 94, n. I-2, p. 8-1 5, fev. 2010.

https://doi.org/I 0.1016/j.jpubeco.2009.1 I.002

BIDDLE, J. E.; HAMERMESH, D. S. Beauty, Productivity, and Discrimination: Lawyers' Looks and Lucre. Journal of Labor Economics, v. 16, n. I, p. I72-201, jan. 1998. https://doi.org/l 0. I086/209886

BORLAND, J.; LEIGH, A. Unpacking the beauty premium: What channels does it operate through, and has it changed over time? Economic Record, v. 90, n. 288, p. 17-32, dez. 2014.

https://doi.org/I0.1 I I I/|475-4932. I209|

DERYUGINA, T.; SHURCHKOV, O. Does beauty matter in undergraduate education? Economic Inquiry, v. 53, n. 2, 940-961, out. 2015a. https://doi.org/I0.1 I I /ecin. I 2 I 52

DERYUGINA, T.; SHURCHKOV, O. (20I5b). Now you see it, now you don't: The vanishing beauty premium. Journal of Economic Behavior \& Organization, v. I 16, p. 331-345, ago. 20 I 5b.

https://doi.org/l0.1016/j.jebo.2015.05.007

DOORLEY, K., SIERMINSKA, E. Myth or fact? The beauty premium across the wage distribution in Germany. Economics Letters, v. 129, p. 29-34, abr. 2015.

https://doi.org/l0.1016/j.econlet.2015.01.033

EAGLY, A. H.; ASHMORE, R. D.; MAKHIHANI, M. G.; LONGON, L. C. What is beautiful is good, but...: A meta-analytic review of research on the physical attractiveness stereotype. Psychological Bulletin, v. II0, n. I, p. 109-128, 1991. Retrieved from www.apa.org/pubs/journals/bul/

FEINGOLD, A. Good-looking people are not what we think. Psychological Bulletin, v. I I I, n. 2, $304-$ 
HAMERMESH, D. S.; ABREVAYA, J. Beauty is the promise of happiness? European Economic Review, v. 64, p. 35I-368, nov. 2013. https://doi.org/10.1016/j.euroecorev.2013.09.005

HAMERMESH, D. S., BIDDLE, J. E. Nber Working Paper Series Beauty and the Labour Market, n. 4518, nov. 1993.

HAMERMESH, D. S.; PARKER, A. Beauty in the classroom: Instructors' pulchritude and putative pedagogical productivity. Economics of Education Review, v. 24, n. 4, p. 369-376, ago. 2005. https://doi.org/l0.10 16/j.econedurev.2004.07.013

JOHNSON, N. D.; MISLIN, A. A. Trust games: A meta-analysis. Journal of Economic Psychology, v. 32, n. 5, p. 865-889, oct. 20। I. https://doi.org/l0.1016/j.joep.201।.05.007

KALICK, S. M.; ZEBROWITZ, L. A., LANGLOIS, J. H.; JOHNSON, R. M. Does human facial attractiveness honestly advertise health? longitudinal data on an evolutionary question. Psychological Science, v. 9, n. I, p. 8-13, jan. 1998. https://doi.org/I0.1। II/I467-9280.00002

KANAZAWA, S.; KOVAR, J. L. Why beautiful people are more intelligent. Intelligence, v. 32, n. 3, p. 227-243, may 2004. https://doi.org/l 0. I0 I6/j.intell.2004.03.003

LANGLOIS, J. H.; KALAKANIS, L.; RUBENSTEIN, a J.; LARSON, A.; HALLAM, M.; SMOOT, M. Maxims or myths of beauty? A meta-analytic and theoretical review. Psychological Bulletin, v. 126, n. 3, p. 390-423, may 2000. https://doi.org/10.1037/0033-2909. I 26.3.390

LANGLOIS, J. H.; RITTER, J. M.; ROGGMAN; L. A., VAUGHN, L. S. Facial Diversity and Infant Preferences for Attractive Faces. Developmental Psychology, v. 27, n. I), p. 79-84, 1991. disponível em: http://citeseerx.ist.psu.edu/viewdoc/download?doi= 10.1 I I.3 19.5468\&rep=rep I \&type=pdf

MENDEZ, J. M.; MENDEZ, J. P. Student inferences based on facial appearance. Higher Education, v. 7I, n. I, p. I-19, jan. 20 I6. https://doi.org/I0.1007/s 10734-0। 5-9885-7

MOBIUS, M. M.; ROSENBLAT, T. S. Why Beauty Matters. American Economic Review, p. 222-235, 2005. https://doi.org/I0.1038/nature 4443

PARRETT, M. Beauty and the feast: Examining the effect of beauty on earnings using restaurant tipping data. Journal of Economic Psychology, v. 49, p. 34-46, aug. 2015.

https://doi.org/l0.1016/j.joep.2015.04.002

PONZO, M.; SCOPPA, V. Professors' beauty, ability, and teaching evaluations in italy. B.E. Journal of Economic Analysis and Policy, v. 13, n. 2, p. 8I I-835, ago. 20 I3. https://doi.org// 0. I 5 5/bejeap-20 I20041

RUFFLE, B. J.; SHUTUDINER, Z. Are Good-Looking People More Employable? Management Science, v. 6I, n. 8, p. 1760-1776, may 2014. https://doi.org/10.1287/mnsc.2014.1927

SALA, E.; TERRANEO, M.; LUCCHINI, M.; KNIES, G. Exploring the impact of male and female facial attractiveness on occupational prestige. Research in Social Stratification and Mobility, v. 3 I, n. I, p. 6981, mar. 2013. https://doi.org/10.1016/j.rssm.2012.10.003 
SÜSSMUTH, B. Beauty in the classroom: are German students less blinded? Putative pedagogical productivity due to professors' pulchritude: peculiar or pervasive? Applied Economics, v. 38, n. 2, p. 23 I-238, sep. 2006. https://doi.org/I 0.1080/00036840500390296

TODOROV, A.; PAKRASHI, M.; OOSTERHOF, N. N. Evaluating Faces on Trustworthiness After Minimal Time Exposure. Social Cognition, v. 27, n. 6, p. 813-833, 2009.

https://doi.org/l0.1521/soco.2009.27.6.813

WILSON, R. K.; ECKEL, C. C. Judging a Book by its Cover: Beauty and Expectations in the Trust Game. Political Research Quarterly, v. 59, n. 2, p. 189-202, jun. 2006.

https://doi.org/l0.1177//06591290605900202

WOLBRING, T.; RIORDAN, P. How beauty works. Theoretical mechanisms and two empirical applications on students' evaluation of teaching. Social Science Research, v. 57, p. 253-272, maio 2016. https://doi.org/l0.1016/j.ssresearch.2015.12.009

WONG, J. S.; PENNER, A. M. Gender and the returns to attractiveness. Research in Social Stratification and Mobility, v. 44, p. II3-123, jun. 20 I6. https://doi.org/I0. I0 I6/j.rssm.20 I6.04.002 